\title{
Hyperbaric oxygen ameliorates worsening signs and symptoms of post-traumatic stress disorder
}

\author{
This article was published in the following Dove Press journal: \\ Neuropsychiatric Disease and Treatment \\ 3 December 2010 \\ Number of times this article has been viewed
}

\author{
Benjamin Eovaldi' \\ Claude Zanetti ${ }^{2}$ \\ 'Department of Medicine, Chicago \\ College of Osteopathic Medicine, \\ Chicago, IL, USA; ${ }^{2}$ Department \\ of Hyperbaric Medicine, Swedish \\ Covent Hospital, Chicago, IL, USA
}

Correspondence: Benjamin Eovaldi 2217 N Cleveland Ave, Chicago, IL 606I4, USA

Tel + I $616566252 \mid$

Fax +16305157134

Email eovaldi@gmail.com

\begin{abstract}
Hyperbaric oxygen therapy at 2.4 atmospheric pressure absolutes for 90 minutes per day ameliorated the signs and symptoms of agitation, confusion, and emotional distress in a 27 -year-old male seven days following a traumatic accident. Hyperbaric oxygen was used to treat the patient's crush injury and underlying nondisplaced pelvic fractures which were sustained in a bicycle versus automobile traffic accident. Its effect on the patient's neuropsychiatric symptoms was surprising and obvious immediately following the initial hyperbaric oxygen treatment. Complete cognitive and psychiatric recovery was achieved by the seventh and final hyperbaric oxygen treatment. We propose that hyperbaric oxygen was effective in improving the patient's neuropsychiatric symptoms by reducing cerebral oxidative stress, inflammation, vasogenic edema, and hippocampal neuronal apoptosis. Further investigation into the use of hyperbaric oxygen as a novel therapy for the secondary prevention of post-traumatic stress disorder that often accompanies postconcussive syndrome may be warranted. We acknowledge that hyperbaric oxygen therapy has been shown to have a strong placebo effect on neurologic and psychiatric diseases.
\end{abstract}

Keywords: hyperbaric oxygen, post-traumatic stress disorder, neuropsychiatric, acute stress disorder

\section{Introduction}

Post-traumatic stress disorder (PTSD) is a global problem that continues to grow as our understanding and use of simple explosive chemistry becomes more prevalent. The general population is now beginning to view PTSD as more of a public health concern, largely because the media has increased their focus on the impact that PTSD has on military personnel returning to the US from service in Iraq. In the civilian population, PTSD following motor vehicle accidents, the most common cause of PTSD, is less likely to be recognized by the general population and medical community than PTSD caused by exposure to military combat. ${ }^{1,2}$ Research and evidence related to the pathogenesis of PTSD suggests that the dysfunction is not merely present in the mind, but that organic changes in the hippocampus and limbic system are also involved. ${ }^{2-4}$ Humans are regularly exposed to painful or difficult situations, and manageable stress is considered to play a positive role in our lives. However, uncontrollable stress is the primary etiologic factor in the development of acute stress disorder and PTSD. ${ }^{2}$

Acute stress disorder and PTSD are the same constellation of symptoms involving intense fear, sleep abnormalities, behavioral changes, and intrusive thought processes that may develop following exposure to a life-threatening event. The duration of disturbance must be between two days and four weeks in acute stress 
disorder and greater than one month in acute PTSD as per the Diagnostic and Statistical Manual of Mental Disorders, fourth edition (DSM-IV) criteria. Because PTSD has been shown to be particularly difficult to treat and has a profound negative impact on the affected patient's quality of life, it is important to recognize the signs and symptoms early in at-risk patients in order to attempt to stop progression from acute stress disorder to PTSD. Several studies have shown hyperbaric oxygen therapy to be effective in preventing or treating acute psychiatric disturbances that are associated with stress and disease. , $^{4,5}$

We suggest that it is reasonable to investigate if hyperbaric oxygen therapy is an effective modality for treating acute stress disorder and if it may serve potentially as secondary prevention of PTSD in patients who have experienced life-threatening trauma resulting in crush injuries because these victims of trauma are at increased risk for developing PTSD, and hyperbaric oxygen is an indicated therapy for their crush injuries. ${ }^{6,7}$ Hyperbaric oxygen therapy has been used with varying degrees of success in treating chronic psychiatric and neurologic diseases and is currently being investigated for the potential treatment of autism, multiple sclerosis, and chronic traumatic brain injury. ${ }^{8}$ Acute neurologic and psychiatric disturbances such as acute traumatic brain injury, lacunar infarcts, acute stress disorder, and acute PTSD may respond better than chronic neurologic and psychiatric conditions to hyperbaric oxygen therapy.

Currently eight of the 13 disorders that hyperbaric oxygen is approved for treating are acute conditions. ${ }^{6,9-12}$ There is strong evidence that hyperbaric oxygen therapy decreases vasogenic edema and inflammation in soft tissue injuries. ${ }^{6}$ The mechanisms involved for hyperbaric oxygen treating soft tissue injuries are related to the extreme elevations in tissue oxygen tensions generated under supra-atmospheric oxygen pressures and are discussed further in this report. Because the brain is an electrical organ and its functional networks and supportive structures are made up of different cells than soft tissues, the mechanisms involved in treating nervous tissue with hyperbaric oxygen are probably also different but may act similarly. ${ }^{13,14}$

\section{Case report}

A fit 27-year-old male presented to the hyperbaric oxygen department in pain and emotional distress seven days following a traumatic accident. His appeared agitated and reported that his symptoms of confusion, intense fear, and intrusive thoughts of the accident were becoming worse each day. The trauma occurred when the patient was cycling through a traffic intersection and was hit directly in the left hip by a car traveling at approximately 30 miles/hour. The collision displaced the patient from his bicycle. His left shoulder impacted and shattered the driver's side windshield. His body continued to roll over the automobile and was launched into the air with a trajectory perpendicular to the vector of the automobile. The patient landed on the road 15 feet away from the site of impact.

Following the trauma, the patient reported a transient loss of consciousness. He was alert, oriented, responded to questions appropriately, and there were no focal neurologic deficits on physical examination. The patient was able to move all four of his extremities. However, he reported severe pain in his left hip upon movement. He was highly agitated and expressed himself with strong emotional language at the scene. Computed tomography (CT) of the head was unremarkable. The only abnormal X-ray finding was a small avulsion fracture of the ankle. The patient's pain in the emergency department at rest was controlled with $60 \mathrm{mg}$ of ketorolac intramuscularly and two tablets of paracetamol three times a day orally. The following day, magnetic resonance imaging (MRI) of the patient's left hip, pelvis, and lumbar spine was performed to look for orthopedic injuries because the patient had a considerable amount of pain with limb movement. MRI of the left hip and pelvis were positive for a large left flank hematoma, several pelvic fractures, and a femoral head contusion.

The patient began experiencing frequent episodes of crying on the day of the accident. He reported recurrent intrusive thoughts which were described as "attempts to piece together or find the details that were missing from the time of being hit until landing in the road". He experienced insomnia associated with nightmares and became progressively more fearful each day following the accident. On the fourth day he initiated the process of re-exposing himself to the stressful situation by riding another bicycle through the streets of Chicago. The patient reported that he was worried that he was experiencing signs and symptoms of PTSD and felt as if he were losing control and did not know what to do.

Prior to the event the patient had not experienced any significant trauma. His medical history was only positive for seasonal allergies for which he occasionally took loratadine. The patient did not have a psychiatric history and denied tobacco, alcohol, and drug use. His family history was positive for breast cancer in his mother and hypertension in his father; there was no family history of psychiatric illness. In spite of having a clean medical and psychiatric history our patient possessed several risk factors for developing acute 
stress disorder and PTSD, ie, sustained accompanying physical injury, a manmade life-threatening event, and a strong emotional reaction to the traumatic event.

Hyperbaric oxygen therapy was initiated to treat the patient's crush injury. After the first hyperbaric oxygen treatment, the patient appeared remarkably less agitated upon exiting the chamber, displayed an appropriate state of arousal, and appeared calm. He reported that his sense of security had returned and that he was able to sleep comfortably without nightmares. The patient's episodes of crying had also resolved. By the seventh treatment, the patient was free from all psychiatric and cognitive symptoms. A psychiatric evaluation and scoring by the clinician-assessment PTSD scale confirmed that the patient was experiencing worsening signs and symptoms of acute stress disorder and that they resolved promptly with hyperbaric oxygen therapy.

\section{Discussion}

We acknowledge that our patient's psychiatric disturbance would have most likely resolved spontaneously without hyperbaric oxygen therapy with more time, because most cases of acute stress disorder do not progress into PTSD. However, our patient's symptoms of confusion and agitation were markedly reduced upon his removal from the hyperbaric chamber following the initial treatment. Because hyperbaric oxygen treatment has been shown to have a strong placebo effect on neurologic and psychiatric diseases it is possible that his condition improved because of the simple expectation of feeling better with treatment. ${ }^{15}$ However, in our case it is not likely that the placebo effect played a significant role in improving our patient's cognitive and psychiatric symptoms because the patient only expected his pelvic injury to improve from hyperbaric oxygen therapy. It is also possible that the effect was because of the pain relief which hyperbaric oxygen treatment provided. Despite the limitations of the study we believe our case is significant because our patient's fear, emotional distress, and agitation were progressively worsening each day post-trauma until the initial hyperbaric oxygen treatment.

Cerebral CT scan in the emergency department was ordered to exclude the possibility of a life-threatening head injury, such as a subdural hemorrhage, which could be missed clinically if the presenting symptoms were subtle. Because the patient did not complain of a headache and focal neurologic abnormalities were not observed during neurologic examinations, MRI of the brain was not ordered by any of the physicians who cared for the patient. Cerebral MRI, magnetic resonance angiography, magnetic resonance spectroscopy, or noninvasive optimal blood flow analysis may have shown subtle findings consistent with the patient's history such as vasogenic edema, diffuse axonal injury, or alteration in cerebral blood flow. ${ }^{16,17}$ Highly sophisticated cerebral imaging will likely play a crucial role, serving as an objective assessment of the efficacy of hyperbaric oxygen in treating neuropsychiatric disorders following traumatic injury as more studies are completed. ${ }^{16-19}$

The pharmacologic mechanisms of hyperbaric oxygen therapy appear to work against more than one pathophysiologic process. We propose that the overall therapeutic effect of hyperbaric oxygen therapy on acute traumatic neuropsychiatric dysfunction is to reduce vasogenic edema and limit hippocampal dysfunction. ${ }^{20-24}$ Following traumatic neurologic injury, it is important to achieve optimal clearing of the proinflammatory cytokine storm associated with acute central nervous system injury. It is well known that reducing cerebral vasogenic edema improves neurologic outcomes following trauma. Oxidative stress and inflammation together disturb laminar blood flow by altering macrophages and $\mathrm{T}$ lymphocyte function which results in cell accumulation and adherence to the endothelium..$^{6,711,12}$ Hyperbaric oxygen therapy generates tremendous tissue oxygen tensions which reverse in vivo electrochemistry to a reductive state and has been shown to prevent cerebral lipid peroxidation following carbon monoxide exposure. ${ }^{11,25-27}$ The anti-inflammatory effects of hyperbaric oxygen may involve reduction of glutathione and suppression of tumor necrosis factor- $\alpha$, interleukin-6, and tissue myeloperoxidase. ${ }^{20,28}$

Damage to the hippocampus and limbic system appears to be important in the development of PTSD, the postconcussive syndrome, and postdepressive syndromes..$^{20,21,24}$ The hippocampus is uniquely positioned among the vascular territories of the posterior cerebral artery, anterior cerebral artery, anterior choroidal artery (a branch of the internal carotid artery), and penetrating branches of the middle cerebral artery. ${ }^{25}$ The rich collateral blood flow to the hippocampus and limbic region is a therapeutic target. Damage to the surrounding microvasculature and endothelium in the injured tissue increases capillary permeability and displaces fluid to the interstitial space. Increased interstitial osmotic pressures force fluid into surrounding cells which results in concomitant intracellular edema. ${ }^{12,24}$ Intra- and extracellular edema together promote hypoxia, cell death, and tissue ischemia. Hyperbaric oxygen therapy counteracts the vasogenic edema complex of edema, hypoxia, and vasodilation by acting as a robust vasoconstrictor., ${ }^{7}, 12$ Vasoconstriction reduces blood flow to edematous tissue and effectively 
reverses the hydrostatic pressure gradient to favor lymphatic and microvasculature fluid reabsorption. Reducing vasogenic edema improves venous outflow and ultimately restores functional vascular flow. ${ }^{6}$

Damage to the hippocampus has been repeatedly implicated as a causal factor in the development of PTSD, the postconcussive syndrome, and postdepressive syndromes. ${ }^{13-15,25,26}$ PTSD is associated with hippocampal atrophy in humans. ${ }^{21,22}$ A proposed mechanism for the hippocampal dysfunction involves the activation of mitochondrial apoptotic pathways mediated via pathophysiologic crosstalk elements, ie, reactive oxygen species, cytochrome c, and caspases. ${ }^{19,24}$ Exposing rats to a single prolonged stress, a proposed method for inducing PTSD in animals, reproducibly kills hippocampal neurons via apoptosis. ${ }^{26,27}$ Preconditioning rats with induced PTSD using hyperbaric oxygen prevented the loss of hippocampal neurons by inhibiting mitochondrial apoptotic pathways. Hyperbaric oxygen preconditioning also improved the animals' neurologic and behavioral outcomes. ${ }^{26,27}$ Preconditioning with hyperbaric oxygen has also been shown to reduce postpump depression significantly following cardiopulmonary bypass..$^{13,14}$ Directing therapy to serve as secondary prevention of PTSD for at-risk patients or patients with early cognitive and psychiatric disturbances following trauma may be useful. There is recent evidence suggesting that the use of morphine in acute trauma care may reduce the risk of developing PTSD following serious injury by blocking memory consolidation mediated via adrenergic pathways. $^{29}$

\section{Conclusion}

Several other case studies have been reported in which hyperbaric oxygen therapy resulted in a dramatic reduction or abolition of psychiatric symptoms in individuals suffering from postconcussive syndrome with and without associated PTSD features. ${ }^{17,30,31}$ Remarkably, hyperbaric oxygen therapy was effective in eliminating psychiatric symptoms when initiated six months to three years post-trauma. We report the first case of hyperbaric oxygen ameliorating the signs and symptoms of acute stress disorder following a life-threatening traumatic injury. We suggest that hyperbaric oxygen therapy was effective in improving our patient's acute psychiatric disturbance by effectively counteracting the pathologic processes of oxidative stress, acute inflammation, vasogenic edema, and hippocampal neuronal apoptosis. Our case may support further investigation into the use of hyperbaric oxygen therapy as a novel treatment for the secondary prevention of PTSD and postconcussive syndromes. ${ }^{29,32}$

\section{Disclosure}

The authors report no conflicts of interest in this work.

\section{References}

1. Olsson A, Phelps EA. Social learning of fear. Nat Neurosci. 2007;10: 1095-1102.

2. Southwick SM. Post traumatic stress disorder. Audio-Digest Internal Medicine. 2007:54.

3. Bossini L, Tavanti M, Lombardelli A, et al. Changes in hippocampal volume in patients with post-traumatic stress disorder after sertraline treatment. J Clin Psychopharmacol. 2007;27:133-135.

4. Milliken CS, Auchterlonie JL, Hoge CW. Longitudinal assessment of mental health problems among active and reserve component soldiers returning from the Iraq war. JAMA. 2007;29:2141-2148.

5. Shi XY, Tang ZQ, Sun D, He XJ. Evaluation of hyperbaric oxygen treatment of neuropsychiatric disorders following traumatic brain injury. Chin Med J. 2006;23:1978-1982.

6. Williams S. The role of hyperbaric oxygen therapy in trauma. Trauma. 2010;12:13-20.

7. Sheffield PJ. Tissue oxygen measurements with respect to soft-tissue wound healing with normobaric and hyperbaric oxygen. Hyperb Oxygen Rev. 1985;6:18-48.

8. Bennett M, Mitchell S, Neuman T, et al. Hyperbaric oxygen therapy and neurological disease. Undersea Hyperb Med. 2010;5:371-373.

9. Bitterman H. Bench-to-bedside review: Oxygen as a drug. Crit Care. 2009;9:205-214.

10. Kudchodkar B, Jones H, Simecka J, Dory L. Hyperbaric oxygen treatment attenuates the pro-inflammatory and immune responses in apolipoprotein E knockout mice. Clin Immunol. 2008;128:435-441.

11. Thom SR. Antagonism of carbon monoxide-mediated brain lipid peroxidation by hyperbaric oxygen. Toxicol Appl Pharmacol. 1990;105: 340-344.

12. Sukoff MH, Ragatz RE. Hyperbaric oxygenation for the treatment of acute cerebral edema. Neurosurgery. 1982;10:29-38.

13. Yogaratnam JZ, Laden G, Guvendik L, Cowen M, Cale A, Griffin S. Hyperbaric oxygen preconditioning improves myocardial function, reduces length of intensive care stay, and limits complications post coronary artery bypass graft surgery. Cardiovasc Revasc Med. 2010; 11:8-19.

14. Alex J, Laden G, Cale A, et al. Pretreatment with hyperbaric oxygen and its effect on neuropsyshometric dysfunction and systemic inflammatory response after cardiopulmonary bypass: A prospective randomized trial. J Thorac Cardiovasc Surg. 2005;130:1623-1630.

15. Vila JF, Balcarce PE, Abiusi GR, Dominguez RO, Pisarello JB. Improvement in motor and cognitive impairment after hyperbaric oxygen therapy in a selected group of patients with cerebrovascular disease: A prospective single-blind controlled trial. Undersea Hyperb Med. 2005;5:341-349.

16. Amin-Hanjani S, Rose-Finnell L, Richardson D, et al. Vertebrobasilar Flow Evaluation and Risk of Transient Ischaemic Attack and Stroke study (VERiTAS): Rationale and design. Int J Stroke. 2010;5: 499-505.

17. Harch PG, Fogarty EF, Staab PK, van Meter K. Low pressure hyperbaric oxygen therapy and SPECT brain imaging in the treatment of blastinduced chronic traumatic brain injury (post-concussive syndrome) and post traumatic stress disorder: A case report. Cases J. 2009;2:6538.

18. Harch PG, Kriedt C, van Meter KW, Sutherland RJ. Hyperbaric oxygen therapy improves spatial learning and memory in a rat model of chronic traumatic brain injury. Brain Res. 2007;1174:120-129.

19. Sukoff MH, Ragatz RE. Hyperbaric oxygenation for the treatment of acute cerebral edema. Neurosurgery. 1982;10:29-38.

20. Wada K, Miyazawa T, Nomura N, et al. Preferential conditions for and possible mechanisms for induction for ischemic tolerance by repeated hyperbaric oxygenation in gerbil hippocampus. Neurosurgery. 2001;49:160-167. 
21. Hedges DW, Allen S, Tate DF. Reduced hippocampal volume in alcohol and substance naïve Vietnam combat veterans with post-traumatic stress disorder. Cogn Behav Neurol. 2003;16:219-224.

22. Lindauer RJ, Vlieger EJ, Jalink M, et al. Effects of psychotherapy on hippocampal volume in out-patients with post-traumatic stress disorder: An MRI investigation. Psychol Med. 2005;35:1421-1431.

23. Li X, Han F, Liu D, Shi Y. Changes of Bax, Bcl-2 and apoptosis in hippocampus in the rat model of post-traumatic stress disorder. Neurol Res. 2010;32:579-586.

24. Wann BP, Bah TM, Boucher M, et al. Vulnerability for apoptosis in the limbic system after myocardial infarction in rats: A possible model for human post-infarct major depression. J Psychiatry Neurosci. 2007;32:11-16.

25. Kotapka MJ, Gennarelli TA, Graham DI, et al. Selective vulnerability of hippocampal neurons in acceleration-induced experimental head injury. J Neurotrauma. 1991;8:247-258.

26. Li JS, Zhang W, Kang ZM, et al. Hyperbaric oxygen preconditioning reduces ischemia-reperfusion injury by inhibition of apoptosis via mitochondrial pathway in rat brain. Neuroscience. 2009;159:1309-1315.
27. Peng Y, Feng SF, Wang Q, et al. Hyperbaric oxygen preconditioning ameliorates anxiety-like behavior and cognitive impairment via upregulation of thioredoxin reductases in stressed rats. Prog Neuropsychopharmacol Biol Psychiatry. 2010;34:1018-1025.

28. Kawamura M, Sakakibara K, Yusa T. Effect of increased oxygen on peripheral circulation in acute, temporary limb hypoxia. J Cardiovasc Surg. 1978;19:161-168.

29. Holbrook TL, Galarneau MR, Dye JL, Quinn K, Dougherty AL. Morphine use after combat injury in Iraq and post-traumatic stress disorder. N Engl J Med. 2010;362:110-117.

30. Hardy P, Johnston KM, de Beaumont L, et al. Pilot case study of the therapeutic potential of hyperbaric oxygen therapy on chronic brain injury. J Neurol Sci. 2007;253:94-105.

31. Wright JK, Zant E, Groom K, Schlegel RE, Gilliland K. Case report: Treatment of mild traumatic brain injury with hyperbaric oxygen. Undersea Hyperb Med. 2009;36:391-399.

32. Larkin M. Can post-traumatic stress disorder be put on hold? Lancet. $1999 ; 18: 1008$
Neuropsychiatric Disease and Treatment

\section{Publish your work in this journal}

Neuropsychiatric Disease and Treatment is an international, peerreviewed journal of clinical therapeutics and pharmacology focusing on concise rapid reporting of clinical or pre-clinical studies on a range of neuropsychiatric and neurological disorders. This journal is indexed on PubMed Central, the 'PsycINFO' database and CAS, and is the official

\section{Dovepress}

journal of The International Neuropsychiatric Association (INA). The manuscript management system is completely online and includes a very quick and fair peer-review system, which is all easy to use. Visit http://www.dovepress.com/testimonials.php to read real quotes from published authors.

Submit your manuscript here: http://www.dovepress.com/neuropsychiatric-disease-and-treatment-journal 